\title{
Blockchain-Based Sugar Inbound Logistics Design Based on the User Wallet Platform Application
}

\author{
Ratna Ekawati ${ }^{1,2^{*}}$, Yandra Arkeman ${ }^{1}$, S Suprihatin ${ }^{1}$, TC Sunarti ${ }^{1}$ \\ ${ }^{1}$ Agro-industrial Engineering Graduate Students IPB University \\ ${ }^{2}$ Staff Industrial Engineering Untirta \\ *Corresponding author. Email: ratna.ti@untirta.ac.id
}

\begin{abstract}
The food and agro supply chain that occurs during the pandemic is the amount of supply available but is constrained by distribution due to a shortage of fleets and resource resources. Perishable agricultural products are wasted, or prices go down due to reduced quality. In this study, the smart contract design that was built was to propose a single wallet specifically for users who could find out all transaction activities carried out by inbound stakeholders, namely production, logistics, and sales in the sugar supply chain at the XY factory. The transactions in the user wallet come from the hash code when the Transaction occurred and the smart contract hash code or algorithm. Because it is a single wallet consisting of several transactions between divisions within the company, the origin of the hash only comes from the user. Meanwhile, inputting the date and volume according to the product, whether it comes from sugar cane or raw sugar, can be traced according to each process such as production, logistics, and also sales by generating a unique smart contract code in the form of a hash. Blockchain-based sugar volume traceability system records all transaction distribution history in an unaltered ledger through smart contract collaboration. An application design presents a transaction response mechanism in verifying the distribution of data entered using a user wallet. All data transaction distribution events are recorded and permanently stored in the Blockchain with a user wallet login. However, all nodes in the supply chain, which are under the supervision of one factory, can participate transparently and reliably.
\end{abstract}

\section{Keywords: Inbound Logistics, Block Chain, Wallet Platform}

\section{INTRODUCTION}

The agricultural sector still lags behind all other sectors in terms of renewable technology, especially information technology. However, pre-harvest and post-harvest processing are still followed by traditional methodologies when tracing, storing, and publishing agricultural data [1]. Agricultural supply chain activities are inseparable from various uncertainties that introduce risks, quantitatively the quantity and quality of products that can affect the flow of components and materials in the supply chain [2]. Sugar is one of the staple foods for the Indonesian people. Even during this pandemic, many people avoid consuming sugar in excess [3].[4]

The problem of food and agro logistics during the current pandemic is the amount of supply available. However, it is constrained by distribution due to lack of fleets, resources, perishable products are wasted, or prices fall due to reduced quality. Therefore, there is unequal distribution, and the local area must make the best use of agricultural products so that regional food security can be maintained. In addition, demand fluctuates with uncertain inputs or processing materials, so many forecasting values are inaccurate.

Tracing product origins across complex supply chains requires a transparent and tamper-resistant metadata infrastructure that is not only trusted by all parties involved but can also adapt to changing environments and regulations. Blockchain technology enables the compilation of reliable traceable data into a single platform. Blockchain enables faster and more accurate decision-making in production and trade, automating various manual activities prone to human error.

This ledger facilitates the sharing of tracking information and promotes networks for multilateral collaboration among supply chain members. The pursuit of transparency and accountability across supply chain 


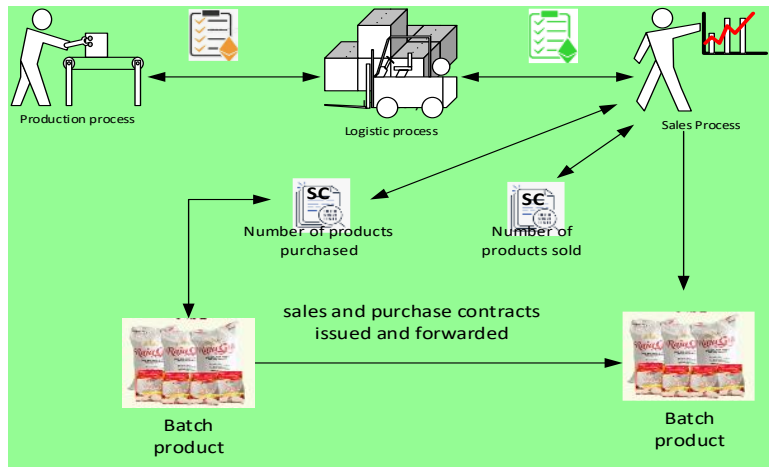

Figure 1. Design system overview.

processes can impact decentralization and automation [4]. As a decentralized, immutable and shared public ledger, the Blockchain consists of additional blocks that include all data transactions and execution results [5]. In a consortium of private Blockchain, only a few trusted nodes are involved in the decision to create a new block. A product traceability system based on Blockchain technology, where all product transfer history is continuously recorded in a distributed ledger using smart contracts, and a chain is formed that can trace back to the source of the product [6].

Blockchain as a platform for executing smart contracts in $\mathrm{SC}$ as transactions, the use of variable distribution control of data transactions in the model allows for state update operations on Blockchain, which in turn, provides automatic information feedback, intrusion detection, and contract execution control [7]. Blockchain technology is an emerging and developing technology worldwide that plays an important role in smart contracts [8]. Smart contracts for verifiable closed bid auctions on the Ethereum Blockchain [9],[10].

Based on the traditional perception, smart contracts target to reduce transaction costs including arbitration and enforcement fees by realizing traceable and immutable transactions using Blockchain technology for distributed databases [11]. Digital wallet is the latest invention of financial technology, which is a very easy and fast means of exchanging payment transactions [12]. Meanwhile, in the supply chain business process, a wallet is needed as a requirement in the form of contract storage between sugar milling divisions as a tool to achieve precision and transparency of transaction distribution data and become a state-of-the-art article in terms of strategy, technology, and data security.

So the smart contract design that was built aims to propose a special wallet for users for all transaction activities carried out in inbound production volume batch activities, namely production, logistics, and sales in the sugar supply chain at factory XY.

The ecosystem boundary is a sugar milling factory where the sugar produced comes from imported raw sugar and sugar cane from farmers. The players who play a role are production, logistics, and sales. Supply chain tracking focuses on the dynamic response of business activities from processing to payments. Conceptually, the depiction of the event-driven model is centered in Figure 1.

Supply chain information tracking can facilitate the flow of information between players. In addition, it is possible to data the number of product volumes between production, logistics, and sales through the further transformation of the built application. This timely response enables business process automation to reduce bottlenecks and time in checking available stock. This application enters the process flow into a new phase, optimizing order time and product inventory information. Each node in the network corresponds to an Ethereum account, representing its identity in the system and deploying smart contracts.

We assume that the sugar produced in the same batch is identical in quality and structure in the sugar production process, and the differences are negligible. Products produced in different batches need to be reregistered [13]. Therefore, this design updates the process of transferring products in batches, and each batch of products uniquely corresponds to a production batch number. They have the same batch number; for products that are not mass-produced, the batch number represents the individual product.

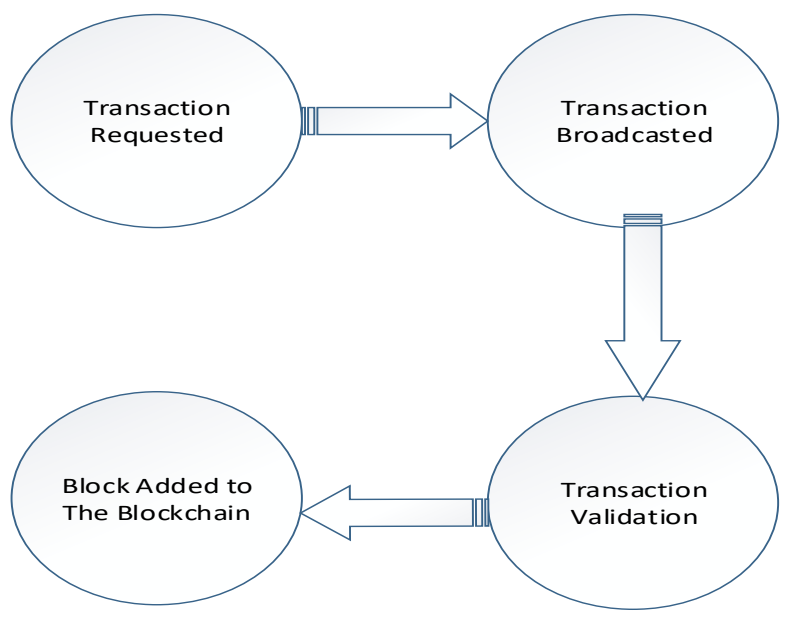

Figure 2. Flow transaction.

Players use smart contracts to connect and communicate with each other. In addition, they provide 
data delivery notifications of the latest updates regarding the status of the process. In this study, certain contracts function as administrators or users who connect other smart contracts. Contracts are deployed to a Blockchain-based platform, in this case, Ethereum, and each contract plays a different role in three main processes. The interactions and relationships between these contracts and their associated sub-processes are described as follows.

In this case, the user logs in using the user and password. Likewise, the wallet used is based on the Metamask wallet. The inputted data is distributed to stakeholders, then the sender, in this case, the production, requests a transaction. The transaction request is announced on a Peer to Peer (P2P) network consisting of nodes. The node will validate the hash entered using an algorithm so that a new smart contract appears in the form of a new hash and so on during data transactions to add new blocks that are bound together.

\section{METHODS}

Non-Blockchain data collection is very time-consuming and expensive. Blockchain technology can automatically track all aspects of production activities such as product origin, quality control metrics, e-mail, and other supply chain data flows that are important to your business. This creates an ideal environment for trading and sharing data. Blockchain creates trust Blockchain is considered the most significant achievement in technology since the birth of the internet.

The new information encryption algorithm is the key that protects data security in supply chain management which was developed in the formal framework for product traceability in supply chain management [14] Any data in a committed transaction eventually becomes immutable. Cryptographically signed historical transaction chains cannot. Cryptographic tools also support data integrity, public access provides data transparency, and each participant potentially has the same ability to access and manipulate the Blockchain. Participants rely on the Blockchain network itself, not on trusted third-party organizations. While procedural languages are typically used to program smart contracts in Blockchain systems, logic-based languages may be an attractive alternative [15] — test for Blockchain using Ethereum with Ropsten Metamask wallet simulation.

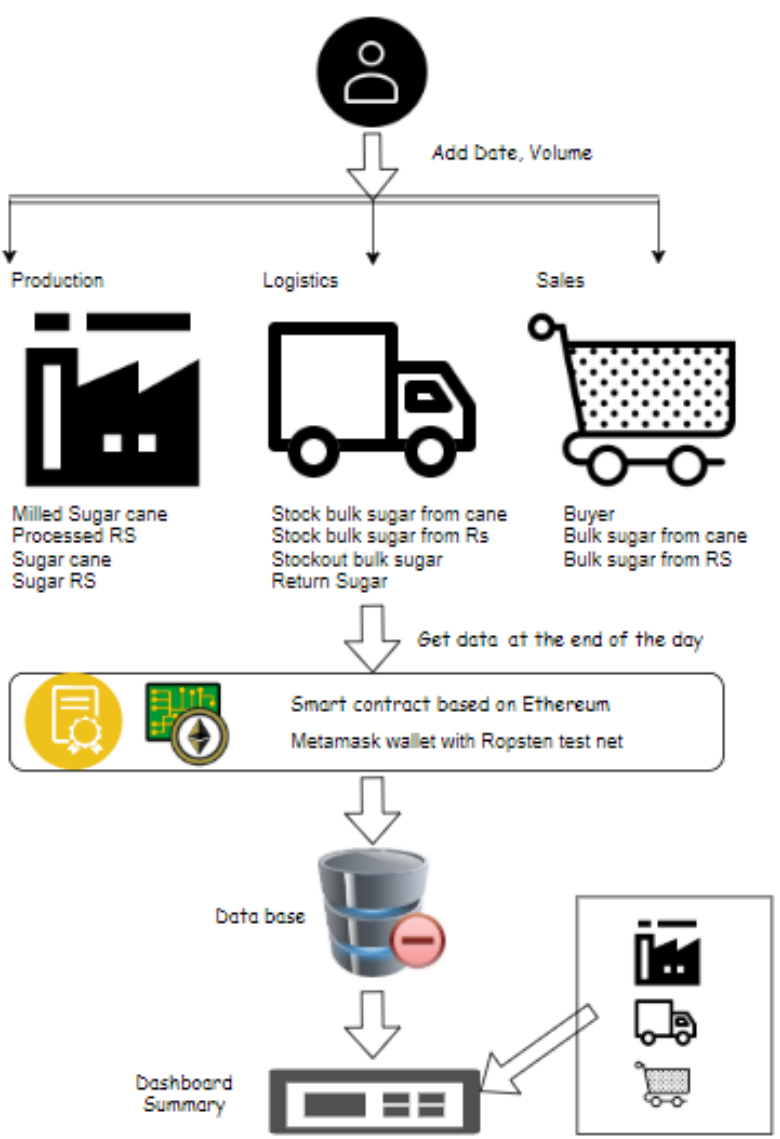

Figure 3. Structure user wallet application based on blockchain.

\section{RESULTS AND DISCUSSION}

We add data based on the date and amount processed in production, logistics, and sales. After that, the data transaction search results will appear on the console in the form of a hash code which we will copy in Etherscan.

The transactions that occur in the user wallet can be seen in fig 6 , where the hash code came from when the Transaction occurred and the smart contract hash code or algorithm. Because this is one wallet, the hash only comes from the user. Meanwhile, the input date and volume according to the product, whether from sugar cane or raw sugar, can be traced according to each process, such as production, logistics, and sales.

Each batch is assigned a contract address to update the product transfer process from the batch in the contract. Each product corresponds to a list of batches, and each batch corresponds to the process of transferring products from this batch. 


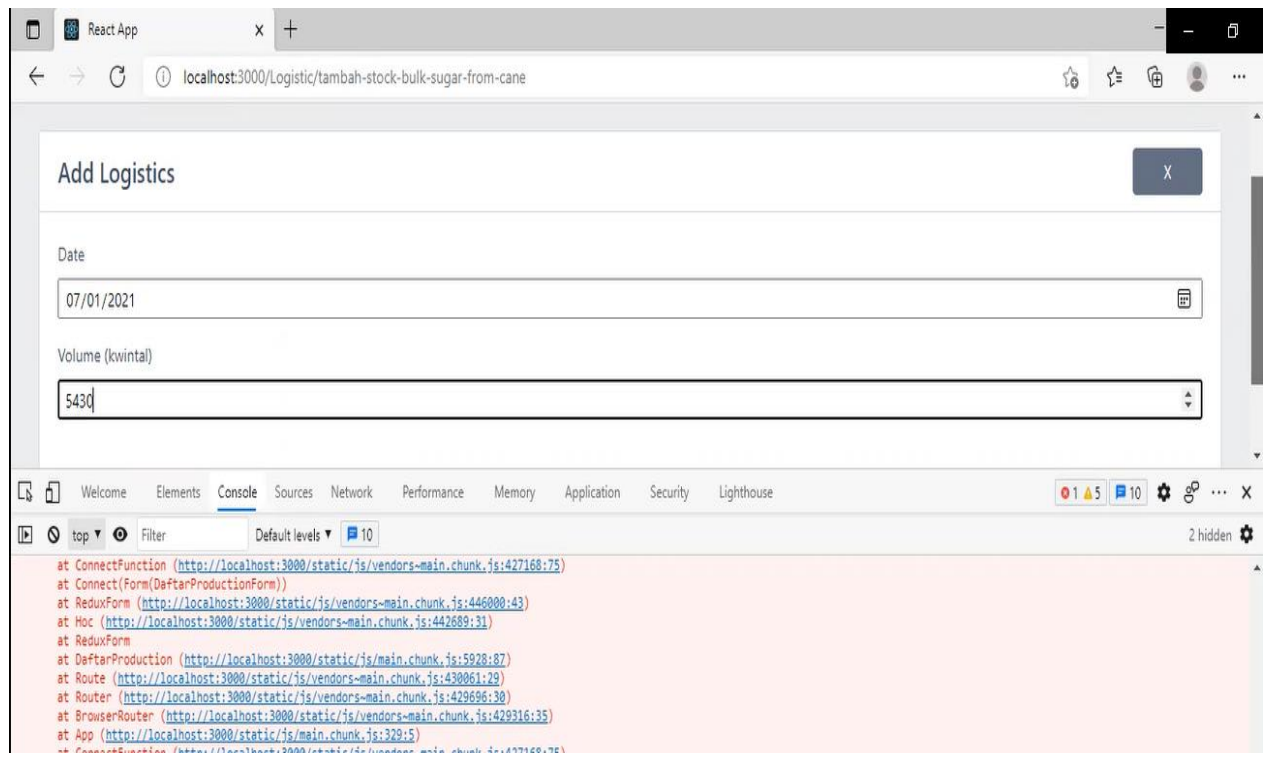

Figure 4. Input data.

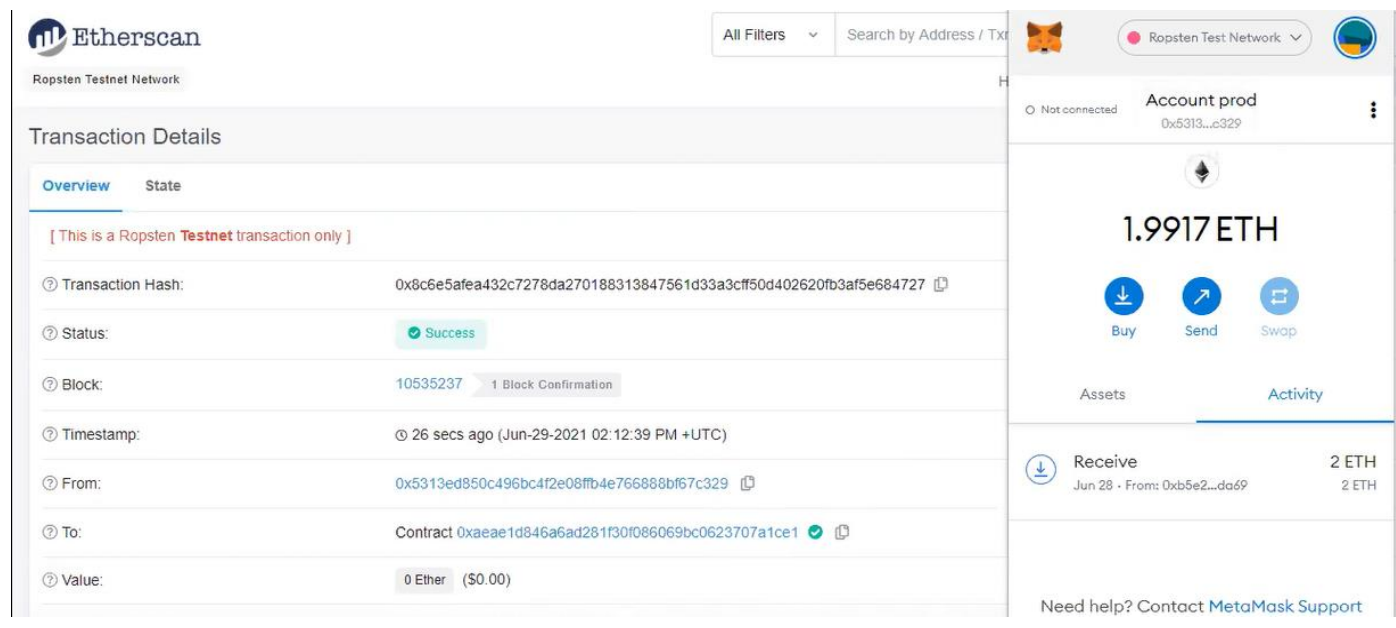

\begin{tabular}{|c|c|c|c|}
\hline \multirow[t]{2}{*}{ (2) Input Data: } & \multicolumn{3}{|c|}{ 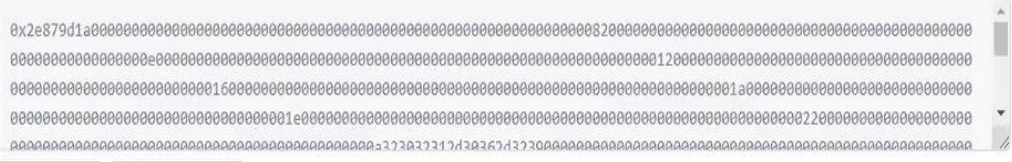 } \\
\hline & Vearlinotits : & De Decose inpul Dath & \\
\hline \multirow[t]{2}{*}{ (2) Input Data: } & & 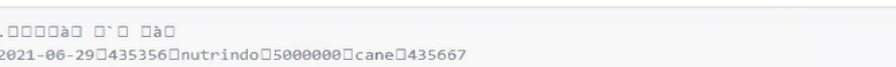 & \\
\hline & & New Input As - S Decode Input Data & \\
\hline
\end{tabular}

Figure 5. Transaction details. 


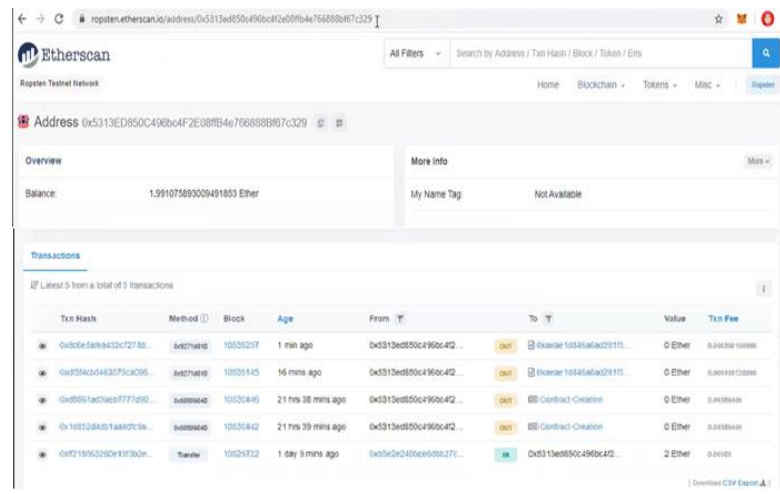

Figure 6. Summary wallet of Transaction.

End consumers can inquire about the transfer history of purchased products with product code and batch number. Also, an authorization list is maintained in each contract, and only accounts added to the authorization list are entitled to renew contract status [16].

The application programming interface (API) used is in middleware because it cannot accept factory authentication, so it is moved out. Middleware is used for two different applications in a unified database, in other words, communicated via API. Bridge two applications from factory application to application Blockchain factory framework integrated into the library, backend localhost 8000 frontend localhost 3000 .

Smart contracts are smart contracts that are a collection of computer code (computer programs) that run on a Blockchain network. In addition, the process of deploying smart contracts to the Blockchain network can only be done once and cannot be changed again. Smart contracts per transaction database entered. Smart contracts can be deployed to the Blockchain only once, so the program code in the smart contracts must be completely flawless and free of bugs.

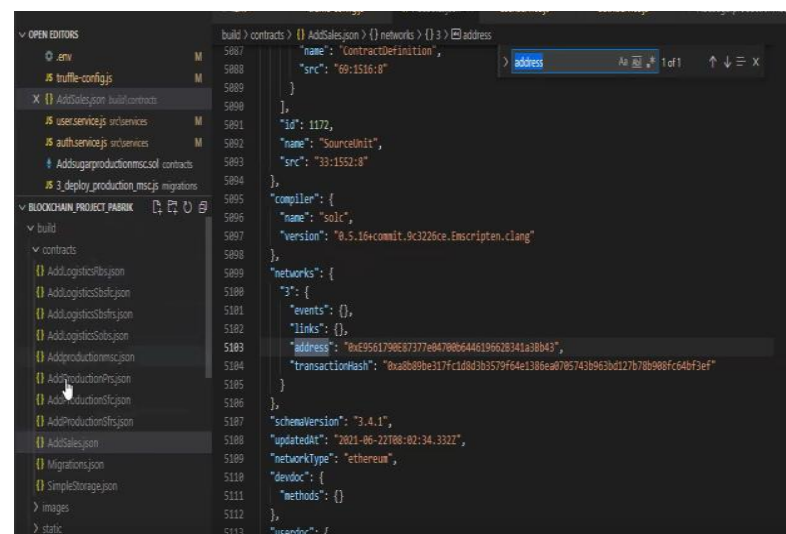

Figure 7. Smart contract algorithm.
Smart contracts are deployed on the Blockchain, and the program code must be audited thoroughly and thoroughly. Audits are carried out to find flaws and vulnerabilities in the program code and fix them.

Blockchain-based sugar volume traceability system records all transaction distribution history in an unaltered ledger through smart contract collaboration. An application design presents a transaction response mechanism in verifying the distribution of data entered using a user wallet. All data transaction distribution events are recorded and permanently stored in the Blockchain with a user wallet login. However, all nodes in the supply chain, which are under the supervision of one factory, can participate transparently and reliably.

We are also building a decentralized application (DApp) based on the truffle framework, deploying smart contracts, and testing contract codes via the Etherscan network with the help of the Ropsten proof of work Metamask wallet running entirely on local memory and implementing our decentralized page interaction interface based on the Ethereum Blockchain platform. The security analysis results show that our system is characterized by data accessibility, tamperproofing, and resistance to man-in-the-middle attacks.

Smart contract formalization has helped in solving and mitigating many of these problems and vulnerabilities. However, as this new domain matures, new vulnerabilities will emerge that will require the combined efforts of the Blockchain research community, cryptography, and formal methods to solve. Therefore, the motivation of our work is to bring this community closer together by summarizing the current achievements and highlighting the open challenges for the future improvement of smart contracts and Blockchain. We expect this work to be an important part of the literature for conducting future empirical analyzes of various smart contract formalization approaches, which could provide further valuable insights into the performance of these approaches as well as highlight key attributes such as accuracy and computational cost.

\section{CONCLUSION}

Based on the results of the smart contract design using the Ethereum platform with the Metamask testnet wallet, namely Ropsten, we conclude that the wallet with the username is for monitoring and controlling the distribution of data transactions starting from the production process, logistics to sales. So the advantage of the designed wallet is that the user can find out who entered the transaction data, transaction time, and also a summary or dashboard of the entire process. So if there is an error or falsification of data, the user can 
find out directly who should be responsible, this is based on the advantages of Blockchain, which are transparent, immutability, and safe from data collectors.

The expected proposal is that this wallet platform application can be applied or become an example of a traceability representative from the company's integration with other sugar mills that are still under the auspices of the company. And the risks that will arise in the future.

\section{ACKNOWLEDGMENT}

This research received financial support from BUDI DN LPDP. I want to thank IPB University and Sultan Ageng Tirtayasa University.

\section{REFERENCES}

[1] T. H. Pranto, A. A. Noman, A. Mahmud, and A. B. Haque, 2021, "Blockchain and smart contract for IoT enabled smart agriculture," PeerJ Comput. Sci., vol. 7, no. March, pp. 1-29.

[2] E. M. Rizqya, K. B. Seminar, and A. Buono, 2017, "Prototype Development of a traceability system for coconut palm sugar supply chain in Indonesia,” Int. J. Res. Sci. Manag., vol. 4, no. 11, pp. 69-76.

[3] A. A. Vazirani, 2021, "COVID-19, an incentive to tackle sugar in hospitals and at home," J. Endocr. Soc., vol. 5, no. 6, pp. 1-4.

[4] S. E. Chang, Y. C. Chen, and M. F. Lu, 2019, "Supply chain re-engineering using blockchain technology: A case of the smart contract-based tracking process," Technol. Forecast. Soc. Change, vol. 144, no. March 2018, pp. 1-11.

[5] A. Bogner, M. Chanson, and A. Meeuw, 2016, "A decentralized sharing app running a smart contract on the Ethereum blockchain," ACM Int. Conf. Proceeding Ser., vol. 07-09-Nove, no. November 2017, pp. 177-178.

[6] S. Wang, D. Li, Y. Zhang, and J. Chen, 2019, "Smart contract-based product traceability system in the supply chain scenario," in IEEE Access, vol. 7, pp. 115122-115133.

[7] A. Dolgui, D. Ivanov, S. Potryasaev, B. Sokolov, M. Ivanova, and F. Werner, 2020, "Blockchainoriented dynamic modeling of smart contract design and execution in the supply chain," Int. J. Prod. Res., vol. 58, no. 7, pp. 2184-2199.

[8] S. R. M. Sekhar, Siddesh G M, S. Kalra, and S. Anand, 2019, "A Study of Use Cases for Smart Contracts Using Blockchain Technology," Int. J. Inf. Syst. Soc. Chang., vol. 10, no. 2, pp. 15-34.
[9] B. L. V. V. Kumar and K. Raja Kumar, 2019,“ Blockchain-based smart contract for sealed--bid auction,” Int. J. Eng. Adv. Technol., vol. 8, no. 6, pp. 628-631.

[10] Y. H. Chen, S. H. Chen, and I. C. Lin, 2018, "Blockchain-based smart contract forbidding system," Proc. 4th IEEE Int. Conf. Appl. Syst. Innov. 2018, ICASI 2018, pp. 208-211.

[11] G. Prause, 2019, "Smart contracts for smart supply chains," IFAC-PapersOnLine, vol. 52, no. 13, pp. 2501-2506.

[12] M. A. Hassan and Z. Shukur, 2019 "Review of Digital Wallet Requirements," in International Conference on Cybersecurity, ICoCSec 2019, no. September, pp. 43-48.

[13] R. Ekawati, Y. Arkeman, Suprihatin, and T. C. Sunarti, 2020, "Design of Intelligent Decision Support System for Sugar Cane Supply Chains Based on Blockchain Technology," in International Conference on Industrial Electrical and Electronics, ICIEE 2020, pp. 153-157.

[14] M. H. Ronaghi, 2020, "A blockchain maturity model in the agricultural supply chain," Inf. Process. Agric., pp. 1-10.

[15] F. Idelberger, G. Governatori, R. Riveret, and G. Sartor, 2016. Evaluation of logic-based smart contracts for blockchain systems, vol. 9718,

[16] A. Singh, R. M. Parizi, Q. Zhang, K. K. R. Choo, and A. Dehghantanha, 2020, "Blockchain smart contracts formalization: Approaches and challenges to address vulnerabilities," Comput. Secure., vol. 88 , p. 101654. 